\title{
Uso do método de Monte Carlo para determinação de custos associados aos estoques de sobressalentes para manutenção
}

Lincon Antonio da Silva

eng_lincon@hotmail.com

Universidade Federal Fluminense (UFF),

Volta Redonda, RJ, Brasil

Nilson Brandalise

nilson 01@yahoo.com.br

Universidade Federal Fluminense (UFF)

Volta Redonda, RJ, Brasil

\begin{abstract}
RESUMO
Este trabalho utiliza o método de Monte Carlo para determinar os custos associados aos estoques de peças de reposição (sobressalentes) para manutenção. Uma planilha eletrônica é utilizada na simulação da aleatoriedade da demanda, permitindo a apuração dos custos para diferentes níveis de estoque, seguindo um modelo de controle e provisionamento de estoques (S, S-1). Esta permite estimar os custos e comparar os resultados frente aos modelos atuais utilizados pela organização explorada neste trabalho, auxiliando ao decisor a determinar qual o nível de estoque onde obtém o menor custo total e proporciona uma maior disponibilidade (menor número de stockouts) no período.
\end{abstract}

PALAVRAS-CHAVE: Tomada de decisão, custos, sobressalentes, simulação de Monte Carlo. 


\section{INTRODUÇÃO}

Todo ativo industrial necessita de manutenção durante seu período de vida a fim de estar disponível e desempenhar uma função definida (GHODRATI, 2006).

Existem diferentes formas de manter estes ativos, dentre as quais necessitarão eventualmente de peças de reposição para substituir componentes desgastados e ou defeituosos por novos. Para isso, peças de reposição sobressalentes importantes devem ser mantidas em estoque e seu inventário devidamente controlado (GHODRATI, 2006).

Godoy et al (2013) considera que a má gestão de peças de reposição que suportam equipamentos críticos leva a impactos consideráveis na estrutura financeira e consequências severas na continuidade operacional. A descontinuidade produtiva de um ativo, por falta de um componente, pode gerar lucros cessantes e grandes prejuízos. No caso, além da perda de produção por máquina parada, soma-se o custo da recuperação do ativo por manutenção corretiva. Essa descontinuidade, atrelada aos altos tempos de suprimento das peças sobressalentes pode, inclusive, levar a ruptura de oferta do produto final, o que possibilita a perda de participação em mercados quando estes apresentam alta competitividade (SANTOS, 2015).

A continuidade de produção e a otimização de custo de sobressalentes são forças contrapostas, nas quais, de um lado, apresenta-se a tendência por geração de despesas e disponibilidade total de materiais para manutenção dos ativos industriais e, de outro, a busca por um estoque mínimo que gere menor custo (SANTOS, 2015).

Determinar o balanço apropriado entre a disponibilidade e custos de estoques é muito desafiador devido às características particulares da demanda de sobressalentes. As demandas são geralmente intermitentes, isto é, ocorrem infrequentemente e com muitos períodos sem nenhuma demanda - o comprimento médio de seu intervalo inter-demanda é grande (TURRINI; MEISSNER, 2017). Adicionalmente os tempos de resposta do ressuprimento são longos (GOMES; WANKE, 2008; TEODORO FILHO et al., 2017; COSTA, 2009).

Nota-se, então, que existe uma dicotomia motivada por interesses divergentes e internos das organizações, em que, os setores de Contabilidade e Finanças olham os volumes de capital imobilizados em prateleiras de estoque e pressionam para reduzi-los. E, em contraposição, os setores da Operação, Manutenção e Produção pressionam por segurança operacional e disponibilidade total de seus equipamentos. No meio dessa divergência, o decisor se depara com seu principal trade-off: manter o equilíbrio entre aumentar a disponibilidade e reduzir custo (SANTOS, 2015).

Este artigo tem como objetivo geral o uso do método de Monte Carlo para determinar os custos associados ao estoque de peças sobressalentes para manutenção, e como objetivos específicos: estudar manutenção; estudar peças sobressalentes; estudar o método de Monte Carlo; definir os níveis de estoque das peças sobressalentes.

A justificativa para este trabalho é utilizá-lo como meio de apoio a decisão, contribuindo para a diminuição da lacuna entre academia e a prática na indústria, uma vez que poucas áreas dentro da administração da produção têm sido tratadas 
de forma tão pouco formal quanto a gestão dos estoques de peças sobressalentes e tem sido raro encontrar empresas brasileiras que utilizem métodos quantitativos formais para apoio à gestão; ao contrário, é comum o uso de métodos empíricos, qualitativos e baseados em intuição (GIANESI; BIAZZI, 2011) e sendo abordada com base em tentativa e erro, sugestões de experiências passadas e recomendações de fabricantes (TEODORO FILHO et al., 2017).

O presente artigo está estruturado do seguinte modo: a seção 1 apresenta-se a introdução, na seção 2 apresenta-se uma revisão da literatura acerca ao tema de dimensionamento de peças sobressalentes pela manutenção e simulação de Monte Carlo. Na seção 3 apresenta-se a metodologia. A seção 4 apresenta exemplo de aplicação em peças sobressalentes de uma indústria intensiva em capital. A seção 5 apresenta-se as análises de dados e principais conclusões e recomendações para trabalhos futuros. Ao final apresenta-se as referências bibliográficas.

\section{REVISÃO BIBLIOGRÁFICA}

Nesta seção são explorados conceitos sobre manutenção, estoques de peças de reposição sobressalentes e os custos associados, bem como o emprego da simulação de Monte Carlo desenvolvida neste artigo.

\section{MANUTENÇÃO}

A manutenção é definida como a combinação de todas as ações técnicas e administrativas, incluindo as de supervisão, destinadas a manter ou recolocar um item em um estado no qual possa desempenhar uma função requerida (ABNT NBR $5462,1994)$.

As atividades de manutenção resultam de ações executadas no dia a dia para prevenir ou corrigir eventuais anomalias ou falhas detectadas nos equipamentos pelos operadores de produção ou pelas equipes de manutenção. Essas ações podem ser uma simples lubrificação, o reparo de uma falha, a substituição periódica de uma peça, uma grande reforma do equipamento ou até mesmo cuidar da sua operação correta (XENOS, 2012).

As ações que, na tentativa de prevenir a ocorrência de falhas, são antecipadas através da substituição de partes do sistema constituem a manutenção preventiva, (CAVALCANTE; ALMEIDA, 2005) enquanto a manutenção corretiva são as ações de reparo feitas depois que a falha ocorreu. As ações preventivas tomadas com base nos resultados de inspeções periódicas, incluindo a medição de parâmetros para monitorar a degradação do equipamento e detectar, de forma quantitativa, sinais de falha ou condições anormais em suas peças e componentes é conhecido como manutenção preditiva (XENOS, 2012).

Comparado com a manutenção corretiva - somente do ponto de vista do custo de manutenção - a manutenção preventiva é mais cara, pois as peças têm que ser trocadas, e os componentes têm que ser reformados antes de atingirem seu limite de vida (XENOS, 2012).

Em compensação, a frequência de falhas diminui, a disponibilidade dos equipamentos aumenta e também diminuem as interrupções inesperadas da 
produção. Ou seja, se considerarmos o custo total, em várias situações a manutenção preventiva acaba sendo mais barata que a manutenção corretiva, porque se tem domínio das paradas dos equipamentos, em vez de ficar sujeito as paradas inesperadas por falhas nos equipamentos (XENOS, 2012).

A manutenção preditiva pode custar mais do que a preventiva por causa do investimento adicional em hardware e software de monitoramento, custo de gerenciamento, ferramentas e treinamentos necessário para estabelecer um programa de monitoramento. No entanto, fornece uma base para diagnósticos de falhas e operações de manutenção e oferece maior confiabilidade do equipamento e um avanço suficiente nas informações para melhorar o planejamento, reduzindo assim o tempo de paradas inesperadas e os custos operacionais (LEE, J; WANG, H.; 2008).

\section{PEC AS SOBRESSALENTES}

Peças sobressalentes são peças intercambiáveis usadas para o reparo ou substituição de peças defeituosas (ARMENZONI et al., 2015).

Gomes e Wanke (2008) relatam que, de modo geral os estoques de peças sobressalentes não podem ser gerenciados pelos modelos ou métodos tradicionais, já que as condições para sua aplicação não são satisfeitas. Outra razão é que a função do estoque de peças sobressalentes é diferente dos estoques tradicionais. Para as peças sobressalentes, os níveis de serviço usualmente são altos, uma vez que os efeitos da falta (stockout) pode ser particularmente crítico e financeiramente notável. Ao mesmo tempo, o custo de aquisição das peças pode ser muito alto, e como consequência o custo do inventário também se torna relevante. Finalmente, a demanda por sobressalentes é extremamente esporádica e de difícil previsão, e, em algumas circunstancias, podem depender da estratégia de manutenção adotada (ARMENZONI et al., 2015).

\section{REQUISITOS DE PEÇAS SOBRESSALENTES PARA MANUTENÇÃO}

Cheng; Yang; Tsao, (2010) reconhecem que o estoque de peças sobressalentes é fortemente dependente das ações de manutenção. Uma distinção deve ser feita entre manutenção preventiva e manutenção corretiva.

A demanda decorrente da manutenção preventiva está programada e é estocástica em relação ao tamanho da demanda, mas determinista no que se refere à chegada da demanda (WANG; SYNTETOS, 2011). A demanda decorrente da manutenção corretiva, após uma falha, é estocástica em relação à chegada do tempo, mas determinista em quantidade (sendo um na maioria dos casos) (WANG; SYNTETOS, 2011).

\section{CUSTOS DE ESTOQUES DE PEÇAS SOBRESSALENTES}

Os principais componentes de custo de estoque aplicados as peças sobressalentes são: os custos de manutenção de estoque, os custos de aquisição, os custos da falta de estoque. Neste estudo, assume-se que a peça substituída não tem valor residual (DURÁN; MACCHI; RODA, 2016). 
Os custos de manutenção de estoque de uma determinada peça sobressalente são proporcionais ao nível médio de estoque em um determinado período, incluindo os custos associados ao manuseio de materiais, uso de espaço, seguros, obsolescência e um determinado valor correspondente ao custo de retenção da unidade - representado em sua maioria pelo custo de oportunidade - (DURAN; RODA; MACCHI, 2016).

Os custos de aquisição contemplam os custos de compra da peça sobressalente e os custos relacionados para colocar uma ordem de compra (processamento, preparação, transmissão e pedido de compra) feita durante um determinado período.

Os custos de falta ocorrem quando a demanda não é atendida pelo estoque (stockout). Este custo pode ser mensurado como a perda de quantidade de produção, proporcional aos tempos de indisponibilidade do ativo físico (DURAN; RODA; MACCHI, 2016). Esta indisponibilidade pode causar a paralisação de uma área de trabalho por dias e até meses, dependendo do tempo de reposição da peça em falta (RIBEIRO, 2007).

\section{PROVISIONAMENTO DE PEÇAS SOBRESSALENTES}

Se o dimensionamento das peças sobressalentes for baseado nas ações preventivas, as compras serão feitas no estilo just-in-time, ou seja, logo antes da sua utilização. Mas a situação atual de muitas empresas impede que isso aconteça. Com um grande número de falhas nos equipamentos, nunca é possível saber com exatidão que peças serão necessárias e quando. Como resultado apenas uma parcela das peças em estoque está ali para atender ações preventivas planejadas (XENOS, 2012) e a grande parcela são destinadas a ações imprevistas (manutenção corretiva) decorrentes de falhas nos equipamentos.

Uma política de controle estoque comumente empregada, consiste em estocar peças sobressalentes para permitir uma pronta substituição em caso de falha, adiando as atividades de reparo e / ou compra somente depois de ter restaurado as operações do sistema. Esta abordagem visa minimizar os efeitos de uma falha, em termos de tempos de inatividade (indisponibilidade). Isto depende da definição de uma quantidade de peças sobressalentes a estocar para suprir a demanda dentro do tempo de reabastecimento, minimizando o custo de estoque (DURÁN; MACCHI; RODA, 2016).

Outros aspetos importantes devem ser considerados quando forem emitidas as solicitações de reposição: o prazo de entrega exigido pelo fabricante ou fornecedor, o custo das peças de acordo com o volume comprado e limites predeterminados geralmente em intervalos de tempos fixos ou quando é atingido uma quantidade mínima em estoque. Estes parâmetros devem ser continuamente monitorados e compor os modelos de controle e provisionamento dos estoques.

Apresentaremos neste projeto quatro modelos comumente utilizados ( $\mathrm{HU}$ et al., 2018):

a) Modelo $(Q, r)$ - é do tipo revisão contínua e lotes fixos. Quando o nível de estoque atingir ou chegar abaixo do ponto de pedido $r$, é encomendada uma quantidade fixa $Q$; 
b) Modelo $(r, S)$ - é do tipo revisão contínua e lotes variáveis. Quando o nível de estoque atinge o ponto de pedido $r$, é encomendada uma quantidade variável elevando a posição de estoque ao nível máximo S;

c) Modelo $(T, S)$ - é do tipo revisão periódica e lotes variáveis. A cada período de duração $T$ é encomendada uma quantidade suficiente para elevar aposição de estoque ao nível máximo S;

d) Modelo (S, S-1) - é do tipo revisão contínua e lotes variáveis. Quando o nível de estoque está abaixo do nível S, é encomendada uma quantidade suficiente para que a posição de estoque atinja o nível máximo $\mathrm{S}$.

\section{MÉTODO DE MONTE CARLO}

Pelo fato de grande parte das decisões administrativas estarem voltadas para o futuro, especificamente na gestão de estoques que requer estimativas das demandas futuras, é imprescindível que se considere as variáveis que afetam o processo de análise como variando aleatoriamente, já que o exercício de previsão está composto pelo risco do resultado não se efetivar (ROGERS; ROGERS; RIBEIRO, 2004). Para que o processo de simulação esteja presente em uma análise basta verificar se alguma variável do problema assume a condição de aleatoriedade. No caso específico da gestão de estoques, a ferramenta da simulação torna-se uma técnica formal e eficiente que nos auxilia em fundamentar as decisões (ROGERS; ROGERS; RIBEIRO, 2004).

Um dos métodos de simulação mais utilizados é o Monte Carlo. A escolha deste método deve-se devido a facilidade de manuseio, confiança dos resultados e grande quantidade de referências teóricas. As etapas básicas para executar uma Simulação de Monte Carlo estão ilustradas na figura 1.

A simulação neste trabalho é empregada considerando a aleatoriedade da demanda com foco em auxiliar o decisor a determinar o custo associado aos estoques com base no número de peças a serem estocadas conforme o modelo de controle e provisionamento de estoques $(S, S-1)$. O modelo (S, S-1) permite gerenciar os estoques de maneira individual: quando uma peça é retirada do estoque, outra é solicitada para manter o estoque de volta no nível prescrito (COSTANTINO et al., 2017).

Figura 1. Processo de simulação de Monte Carlo.

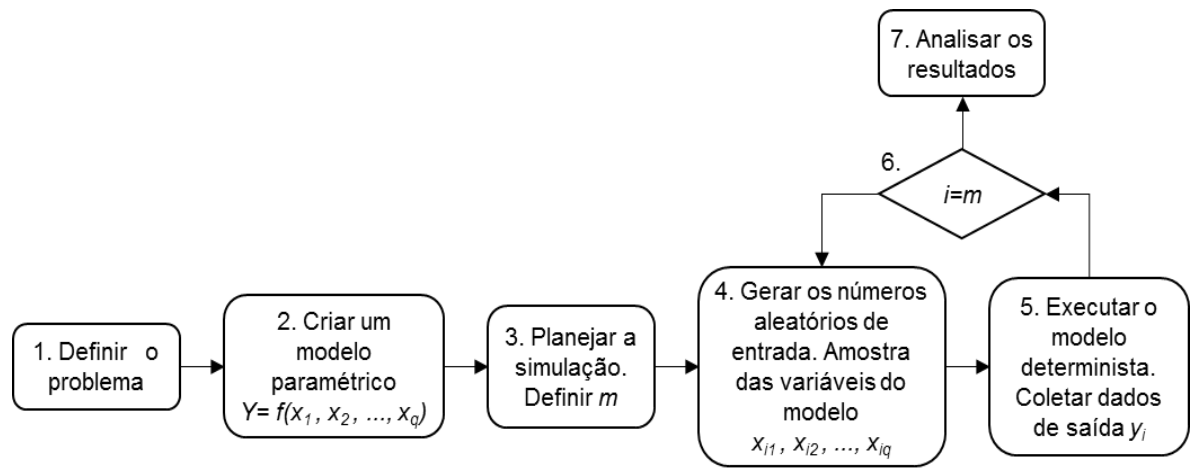

Fonte: adaptado de (O'CONNOR, PATRICK D. T.; KLEYNER, ANDRE, 2012). 
O'Connor e Patrick (2012) descrevem as etapas para aplicação da simulação de Monte Carlo conforme abaixo:

a) Etapa 1: Defina o problema e os objetivos gerais do estudo. Avalie os dados disponíveis e as expectativas de resultados;

b) Etapa 2: Defina o sistema e crie um modelo paramétrico, $y=f\left(x_{1}, x_{2}, \ldots\right.$, $\left.x_{q}\right)$;

c) Etapa 3: Planeje a simulação. As variáveis aleatórias de interesse precisam ser coletadas, tais como as distribuições de probabilidade para cada uma das entradas. O número de simulações $\mathrm{m}$ é afetado pela complexidade do modelo e pela precisão desejada dos resultados. Podemos estimar $\mathrm{m}$ para alcançar certo nível de confiança nas estatísticas da saída simulada calculando o erro padrão da média da distribuição com base no teorema do limite central e na estimativa dos limites de confiança da distribuição normal, através da equação 1 :

$$
\operatorname{Er}(\mu)=\frac{Z \alpha / 2 \times \sigma}{\sqrt{m}} \quad \text { Equação (1) }
$$

Onde: $\operatorname{Er}(\mu)$ erro padrão em relação à média; $Z_{\alpha / 2}$ é o estatístico padrão da distribuição normal para um determinado limite de confiança; $\sigma$ é o desvio padrão; e m é o número de simulações.

A equação 1 tem aplicações limitadas porque o valor de $\sigma$ não é conhecido e só pode ser assumido a priori ou estimado após a primeira simulação.

d) Etapa 4: Gerar um conjunto de entradas aleatórias, $x_{i 1}, x_{i 2}, \ldots, x_{i q} .0$ procedimento de simulação de Monte Carlo requer a capacidade de amostragem de distribuições arbitrárias através da geração uma variável aleatória uniformemente distribuída no intervalo [0; 1$]$.

e) Etapa 5: Execute o modelo de sistema determinístico com o conjunto de entradas aleatórias. Avalie o modelo e armazene os resultados como yi.

f) Etapa 6: Repita as etapas 4 e 5 para $\mathrm{i}=1$ até $\mathrm{m}$.

g) Etapa 7: Analise as estatísticas dos resultados, intervalos de confiança, histogramas, distribuição de melhor ajuste ou qualquer outra medida estatística.

\section{METODOLOGIA}

Nesta seção é apresentado a metodologia empregada e os procedimentos de simulação de Monte Carlo desenvolvida.

\section{CARACTERIZAÇÃO DO MÉTODO DE PESQUISA}

Essa pesquisa pode ser classificada como aplicada, por seu interesse prático na resolução de um problema real existente na definição dos níveis de estoques de sobressalentes. Quanto aos seus objetivos, essa pesquisa pode ser classificada como exploratória, proporcionando maior familiaridade com o problema e análise de exemplos que estimulem a sua compreensão. Quanto a abordagem, essa pesquisa pode ser classificada como quantitativa, visto que requer o uso de 
recursos e de técnicas estatísticas na análise de dados históricos de consumo. 0 método utilizado é a modelagem e simulação, haja vista a utilização de técnicas de tomada de decisão aplicadas a um sistema real, determinando-se como este sistema responderá a modificações que lhe são propostas (TURRIONI; MELLO, 2012).

\section{CARACTERIZAÇÃO DA ORGANIZAÇÃO}

A organização foco deste estudo faz parte de uma empresa de capital intensivo situada no sul fluminense. A organização atualmente tem mais de 230.000 itens sobressalentes cadastrados para manter seus equipamentos e instalações. Destes, cerca de 30.000 itens possuem saldo regular em estoque. A grande maioria destes itens (61\%) são itens de baixíssimo giro (slow movings) com consumo inferior a 1 peça / ano nos últimos 5 anos e de grande importância para organização. Estes são considerados como críticos, pois em caso de falha apresentam grande impacto na produção, qualidade, segurança dos colaboradores ou ao meio ambiente e devem ser substituídos rapidamente de forma a minimizar a indisponibilidade do equipamento ao qual pertencem. O custo de manutenção dos estoques apurado no último ano é de $19,5 \%$, considerando-se um custo de oportunidade de $13,5 \%$.

Por questões de sigilo as informações de custos associados aos sobressalentes foram encobertas levando-se em conta a razão entre os custos de aquisição e os custos de falta / dia.

\section{COLETA E ANÁLISE DOS DADOS}

A peça sobressalente selecionada para análise no presente artigo foi selecionada por conveniência. As informações de custos de aquisição, prazos para ressuprimento (leadtime) e o histórico de consumo mensal - do período de janeiro de 2014 a dezembro de 2017 - foram coletados a partir do sistema ERP (Enterprise Resource Planning) da organização. As informações de consumo retratam o atendimento da demanda pelo estoque e não há a distinção se estas demandas foram oriundas de ações imprevistas decorrentes de falhas de equipamentos (demanda estocástica ou casual) ou de demandas concretas de acordo com o plano manutenção (demanda determinística).

Os custos de falta foram estimados considerando a perda de produção de um dos equipamentos mais importantes do processo produtivo (gargalo de produção) no período de ressuprimento do material.

\section{APLICAÇ̃̃O DO MÉTODO DE MONTE CARLO}

\section{Definição do problema}

O problema de decisão consiste em determinar quantas peças devem ser mantidas em estoque (daqui em diante considerado como Estoque de Segurança) de acordo com os critérios: custo total de estoque $\mathrm{x}$ falta do sobressalente (stockout). O modelo tem como objetivo entender o impacto da incerteza e as 
consequências de diferentes decisões de qual o nível de estoque que obtém o menor custo total e proporciona uma maior disponibilidade (menor número de stockouts) no período.

\section{HISTÓRICO DE CONSUMO}

Preliminarmente faz-se necessário conhecer como é o comportamento da demanda para um determinado item a partir do histórico de consumo. Neste artigo optamos por ajustar uma curva não paramétrica a este comportamento para estimarmos a demanda, uma vez que a característica de consumo de itens slow movings na organização foco deste trabalho carece de relativa ausência de informações de conhecimento a priori a respeito da forma da função que está sendo estimada.

\section{Previsão da demanda}

Com os dados de consumo mensal, elabora-se o histograma simples, e a frequência relativa acumulada será empregada para simular a demanda. Uma planilha eletrônica foi elaborada para simular as demandas num período de 12 meses.

\section{Reposição do estoque}

Adotando-se modelo de controle e provisionamento dos estoques de estoques (S, S-1) são determinados os níveis de estoques a partir da definição do estoque de segurança ( $S$ ). Considera-se que uma nova encomenda é emitida sempre que o nível de estoque está abaixo do estoque de segurança (S), em quantidade suficiente para que a posição de estoque atinja o nível máximo S. A encomenda será atendida considerando um prazo fixo de ressuprimento da peça sobressalente em situação normal e em emergência (caso ocorra a ruptura).

\section{Cálculo dos custos associados aos estoques}

São calculados os custos de estoques considerando a quantidade de peças à ressuprir (custos de aquisição), a parcela correspondente ao período em que o item fica armazenado (custos de armazenagem acrescido do custo de oportunidade) e em caso de ruptura - stockout - os custos da falta até o ressuprimento em regime de emergência para o item.

\section{SIMULAÇ̃̃O EM PLANILHA}

A simulação de Monte Carlo foi desenvolvida em planilha eletrônica Microsoft Excel ${ }^{\circledast}$, versão 2016, largamente utilizada por organizações que as empregam na realização de suas atividades administrativas, tais como controle de folhas de pagamento, finanças, etc. Optou-se em utilizar recursos da Microsoft Office ${ }^{\circledR}$ pois os usuários têm condições de rapidamente se adaptarem ao uso do aplicativo de otimização aqui proposto, sem que incorram em custos com aquisição, 
implantação e treinamento de ferramentas proprietárias, para este fim (SILVA FILHO; CEZARINO; RATTO, 2009).

A Figura 2 ilustra as etapas do processo de simulação empregado.

Figura 2. Processo de simulação de Monte Carlo utilizado.

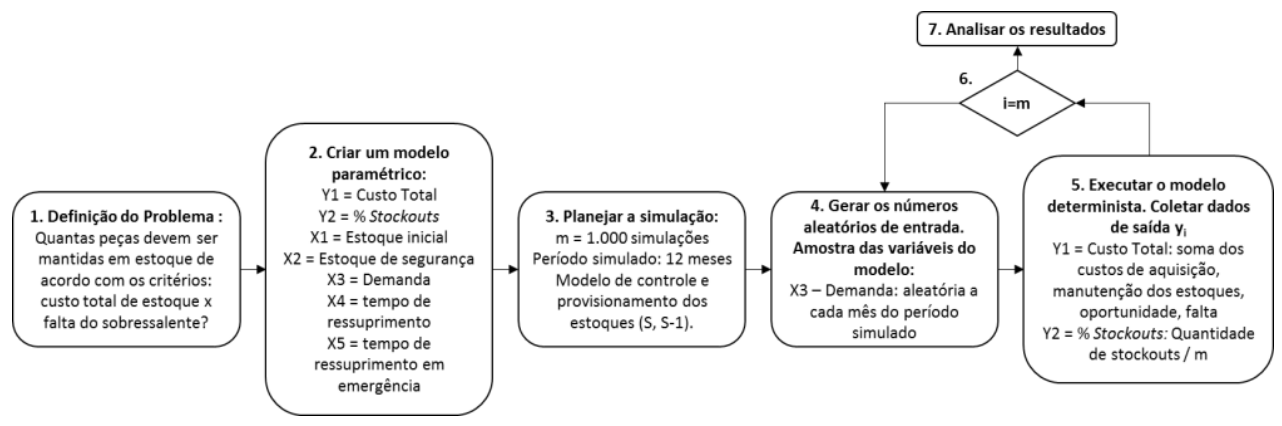

Fonte: Elaborado pelos autores (2018).

Para as simulações, o decisor informa o estoque inicial (na organização foco do trabalho é o saldo atual do item em estoque) e o estoque segurança conforme definição do problema e são coletadas as demais informações da peça sobressalente de interesse.

Inicialmente foram realizadas 1.000 simulações para determinar como será o comportamento da demanda ao longo de 12 meses, os custos associados à dinâmica dos estoques a partir do estabelecimento do estoque de segurança e de um estoque inicial, segundo modelo de controle e provisionamento dos estoques $(S, S-1)$.

A demanda $\left(D_{n}\right)$ é simulada gerando-se um número aleatório através da função $=$ ALEATÓRIO(). 0 resultado é um número entre 0 e 1 (A) que quando comparado com a frequência relativa acumulada do item gera a quantidade demandada.

Para o primeiro período $\left(n_{1}\right)$, o estoque inicial $\left(E I_{1}\right)$ é o informado pelo decisor. Posteriormente a demanda é simulada $\left(D_{1}\right)$ e é apurado o saldo no período $\left(B_{1}=E I_{1}\right.$ $\left.D_{1}\right)$. Caso o saldo em estoque $\left(B_{1}\right)$ for menor que o estoque de segurança $(S)$ gerase uma encomenda de $\left(S-B_{1}\right)$ peças para entrega em situação normal conforme o tempo de ressuprimento $\left(L_{N}\right)$ para $\left(n_{1}+L_{N}\right)$ período. Caso ocorra uma ruptura de estoque $\left(B_{1}<0\right)$ encomenda-se $\left(S-B_{1}\right)$ peças para entrega em situação de emergência conforme o tempo de ressuprimento em emergência $\left(L_{E}\right)$ para $\left(n_{1}+L_{E}\right)$ período.

Para os demais períodos ( $\mathrm{n}_{\mathrm{i}}$, onde i varia de 2 até 12$)$, o estoque inicial $\left(E I_{n}\right)$ é igual ao saldo em estoque do período anterior $\left(B_{n-1}\right)$. Posteriormente a demanda é simulada $\left(D_{n}\right)$. Observa-se se chegarão encomendas - pedidos $\left(P_{n}\right)$ - e é apurado o saldo no período $\left(B_{n}=E I_{n}-D_{n}+P_{n}\right)$. Caso o saldo em estoque $\left(B_{n}\right)$ for menor que o estoque de segurança $(S)$ gera-se uma encomenda de $\left(S-B_{n}\right)$ peças para entrega em situação normal para $\left(n_{n}+L_{N}\right)$ período se não houver encomendas ainda não entregues. Caso ocorra uma ruptura de estoque $\left(B_{n}<0\right)$ encomenda-se $\left(S-B_{n}\right)$ peças para entrega em situação de emergência para entrega em situação de emergência para $\left(\mathrm{n}_{\mathrm{n}}+\mathrm{L}_{\mathrm{E}}\right)$ período. 
Os custos de aquisição apurados consideram o produto entre o custo unitário do item pelo total de encomendas - $\left(P_{n}\right)$ - entregues para o período de 12 meses de simulação. Os custos de manutenção foram ponderados pela existência de saldo por período $\left(B_{n}\right)$ pelo custo de manutenção dos estoques por mês. Os custos de oportunidade foram obtidos de maneira análoga, ponderando pelo custo de oportunidade por mês.

O custo da falta depende da dinâmica dos estoques e é calculado pela quantidade de stockout nos 12 meses de simulação pelo custo da falta/dia vezes o tempo de ressuprimento em emergência $\left(L_{E}\right)$.

\section{LEVANTAMENTO DE DADOS}

Nesta seção são apresentados os dados necessários a simulação de Monte Carlo em planilha eletrônica.

\section{SELEÇÃO DA PEÇA SOBRESSALENTE}

Os custos considerados ao sobressalente selecionado são: razão entre os custos de aquisição e os custos de falta / dia: 1,552; custo de oportunidade por mês $\left(C_{0}\right): 1,06 \%$; Custo de Manutenção dos estoques por mês $\left(C_{M}\right): 0,49 \%$. Os tempos de ressuprimento (leadtime) considerados em situação normal $\left(L_{N}\right)$ e emergência ( $L_{E}$ ) são 6 e 1 mês (es), respectivamente. Por questões de confidencialidade o custo unitário considerado é de $\mathrm{R} \$ 1$.

Tabela 1. Estatística descritiva do histórico de consumo da peça selecionada

\begin{tabular}{cc} 
Período (meses) & Valor \\
Média (unidades/período) & 60 \\
Desvio (unidades / período) & 0,25 \\
Demanda total (unidades) & 0,63 \\
Máxima Demanda (unidades) & 15 \\
Mínima Demanda (unidades) & 4 \\
Demanda Média (unidades) & 1 \\
No de Procuras & 1,3 \\
Intervalo entre procuras (meses) & 12 \\
Coeficiente de Variação & 5,0 \\
\hline
\end{tabular}

Fonte: Elaborado pelos autores (2018).

\section{HISTÓRICO DE CONSUMO}

O histograma observado e a frequência relativa acumulada utilizada para simular a demanda estão dispostos na figura 3. 
Figura 3. Histograma e frequência relativa para os dados de consumo do sobressalent

\section{Histograma - Histórico de Consumo}

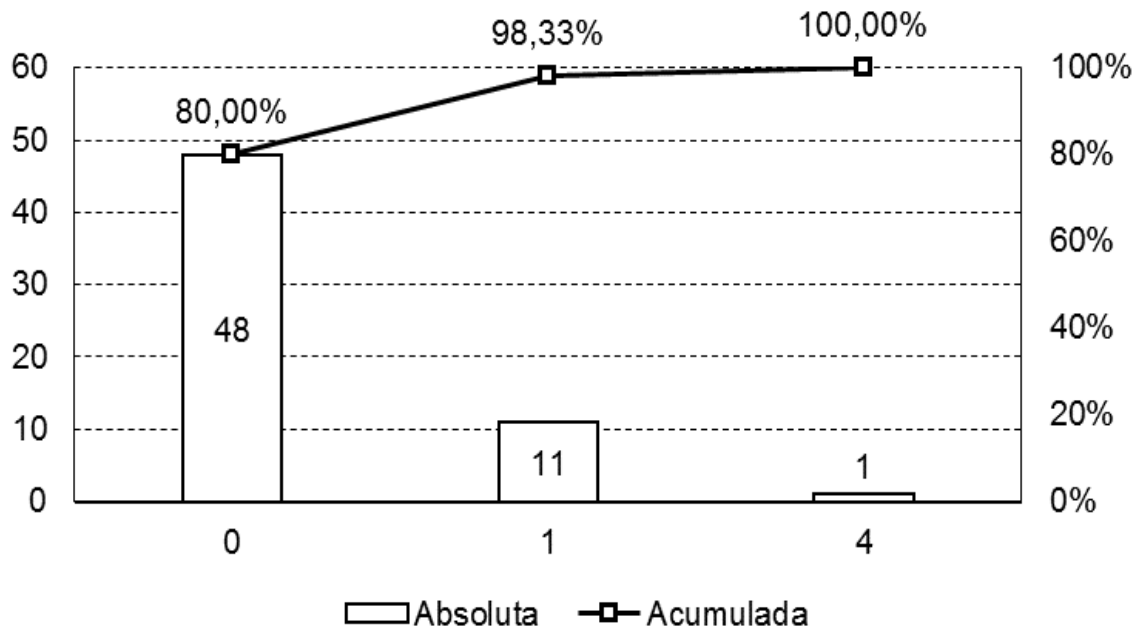

Fonte: Elaborado pelos autores (2018).

\section{ANÁLISE DE DADOS}

Consideramos 5 rodadas de simulações com o estoque de segurança ES variando de 0 a 4 peças e o estoque inicial $\mathrm{El}_{1}$ de 0 peças. Os histogramas dos custos totais simulados para $\mathrm{O} E S=0$ e 4 são apresentados nas figuras 4 a 5 respectivamente.

Figura 4. Histograma e frequência relativa para os custos simulados do sobressalente, considerando o estoque de segurança $S=0$ peças e o estoque inicial $\mathrm{El}=0$ peças.

Histograma, $\mathrm{S}=0 ; \mathrm{El}=0$

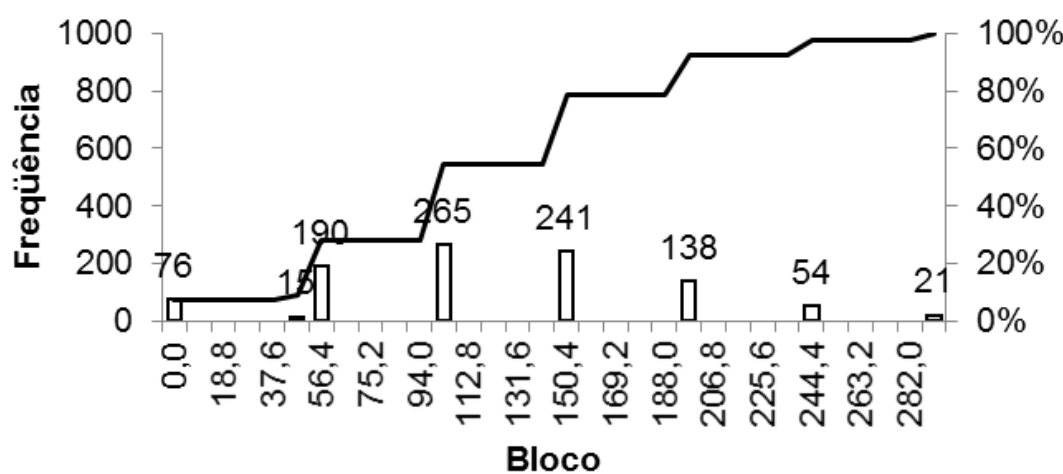

•

Fonte: Elaborado pelos autores (2018). 
Figura 5. Histograma e frequência relativa para os custos simulados do sobressalente, considerando o estoque de segurança $S=4$ peças e o estoque inicial $\mathrm{EI}=0$ peças.

Histograma, $\mathrm{S}=4 ; \mathrm{El}=0$

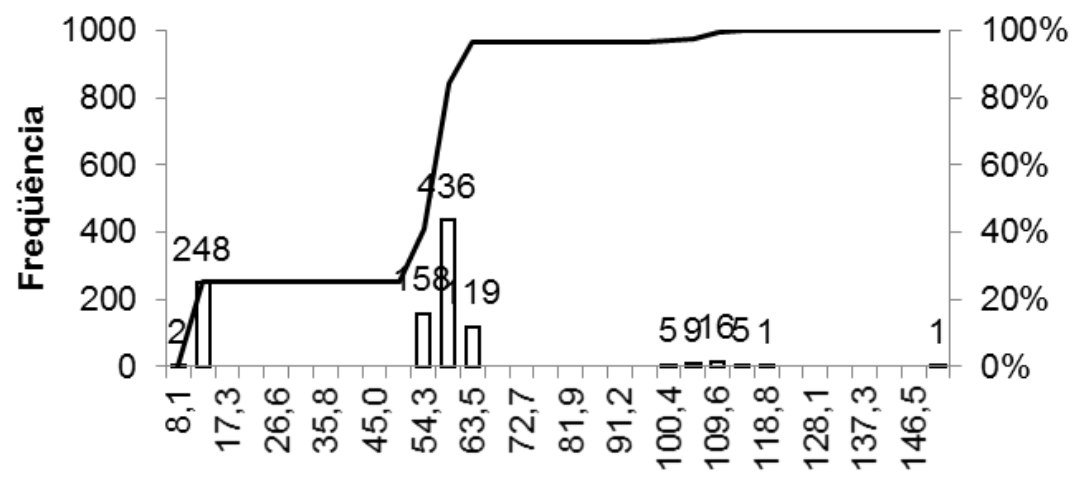

Bloco

$\square$ Freqüência — $\%$ cumulativo

Fonte: Elaborado pelos autores (2018).

Nota-se que a distribuição das frequências acumuladas no histograma (\% cumulativo) possuem forma assimétrica ou não - Normal por causa da distribuição não paramétrica utilizada para a previsão de demanda na simulação.

Tabela 2. Estatística descritiva para diferentes níveis de estoque de Segurança S.

\begin{tabular}{cccccc} 
Média & 19 Rodada & 2a Rodada & 3a Rodada & 4 Rodada & 5a Rodada \\
Desvio Padrão & 114,7 & 58,3 & 47,0 & 47,9 & 46,3 \\
Coeficiente de & 66,8 & 38,0 & 29,8 & 25,9 & 24,0 \\
Variação & 0,6 & 0,7 & 0,6 & 0,5 & 0,5 \\
Mínimo & 0,0 & 2,0 & 4,1 & 6,1 & 8,1 \\
1o Quartil & 47,6 & 49,6 & 49,8 & 51,1 & 41,1 \\
Mediana & 95,1 & 50,6 & 52,0 & 54,2 & 56,3 \\
3o Quartil & 144,9 & 98,2 & 53,1 & 55,2 & 57,3 \\
Máximo & 291,4 & 244,9 & 153,0 & 155,1 & 151,1 \\
Erro & $3 \%$ & $3 \%$ & $3 \%$ & $3 \%$ & $3 \%$ \\
\hline
\end{tabular}

Fonte: Elaborado pelos autores (2018).

A tabela 2 apresenta-se a estatística descritiva para níveis de estoque de segurança $S$ variando progressivamente de 0 peças (1 $\underline{a}$ Rodada) a 4 peças (5a Rodada) mantendo o estoque inicial El de 0 peças. Observa-se que há dispersão dos valores da média e desvio padrão do custo total, com coeficientes de variações maiores que 0,5. Notar, conforme ilustrado por Armenzoni et al. (2015) que a demanda extremamente esporádica e de difícil previsão são fatores contribuintes destes desvios, com iterações com nenhuma demanda no período. 
Tabela 3. Comparativo dos custos simulados para diferentes níveis de estoques.

$\begin{array}{ccccccc}\begin{array}{c}\text { Quantidad } \\ \text { e de peças } \\ \text { (S) }\end{array} & \begin{array}{c}\text { Aquisiç } \\ \text { ão }\end{array} & \begin{array}{c}\text { Manutenção } \\ \text { dos } \\ \text { estoques }\end{array} & \begin{array}{c}\text { Oportunidade } \\ \text { Falta }\end{array} & \begin{array}{c}\text { Total } \\ \text { \% Stock- } \\ \text { outs }\end{array} \\ 1 & 2,7 & 0,0 & 0,0 & 112,0 & 114,7 & 94 \% \\ 2 & 4,0 & 0,0 & 0,1 & 66,5 & 58,3 & 81 \% \\ 3 & 5,5 & 0,1 & 0,2 & 54,7 & 47,0 & 75 \% \\ 4 & 7,4 & 0,1 & 0,3 & 51,2 & 47,9 & 78 \% \\ & 9,0 & 0,2 & 0,4 & 48,9 & 46,3 & 75 \%\end{array}$

Fonte: Elaborado pelos autores (2018).

Os resultados dos custos médios obtidos a partir das simulações estão descritos na tabela 3 . O custo total de não manter peças em estoque (0 peças) é financeiramente notável conforme observado por que conforme Gomes e Wanke (2008). Ressalta-se que maiores quantidades de peças sobressalentes em estoque fazem com que os custos associados diminuam. Isto ocorre principalmente devido a parcela do custo da falta decorrentes de stockouts no período observado. No entanto, o custo da falta tem caráter probabilístico, isto é, sofre com o efeito da incerteza e é por vezes tolerado. A soma dos custos de aquisição, manutenção dos estoques e oportunidades indicam que maiores quantidades de peças em estoque incorrem em maior capital a ser imobilizado sem a contrapartida da redução da falta do sobressalente - \% stockouts.

\section{CONCLUSÃO}

Este trabalho utiliza de abordagens de simulação pelo método de Monte Carlo para a determinação dos custos associados as peças sobressalentes para diferentes níveis de estoque, seguindo um modelo de controle e provisionamento de estoques (S, S-1). Na formulação do problema considera-se apenas a variabilidade da demanda e os seus efeitos na determinação dos custos como auxílio ao trade-off explicito por Santos (2015): aumentar a disponibilidade de peças sobressalentes e reduzir os custos de estoques.

Há evidencias de que estimar os custos é uma aproximação razoável quando comparamos os resultados frente aos modelos atuais utilizados pela organização explorada. No entanto o emprego de métodos quantitativos formais para apoio à gestão de estoques traz vantagens quando comparados aos métodos tradicionais de gestão de estoques e métodos empíricos, qualitativos e baseados em intuição, pois torna-se possível determinar parâmetros de gestão individualizados para cada item, utilizando técnicas quantitativas eficazes conforme explorado por Gianesi e Biazzi (2011).

Foram observadas limitações do trabalho com relação a adoção de custos de aquisição, custos da falta e dos tempos de ressuprimento como determinísticos. Desta forma reconhecem que para trabalhos futuros, estes fatores devam ser considerados a margem da aleatoriedade. 


\title{
USE OF THE MONTE CARLO METHOD FOR DETERMINING COSTS OF SPARE PARTS FOR MAINTENANCE
}

\begin{abstract}
This work uses the Monte Carlo simulation method to determine the costs associated with the stock of spare parts for maintenance. A spreadsheet is used to simulate the randomness of demand, allowing the calculation of costs for different levels of inventory, following a model of inventory control and provisioning $(\mathrm{S}, \mathrm{S}-1)$. This allows estimating the costs and comparing the results against the current models used by the organization explored in this work, helping the decision maker to determine which inventory level obtains the lowest total cost and provides a greater availability (fewer stockouts) in the period.
\end{abstract}

KEYWORDS: Decision-making, costs, spare-parts, Monte Carlo Simulation. 


\section{REFERÊNCIAS}

ABNT NBR 5462:1994. Confiabilidade e mantenabilidade - Terminologia. Associação Brasileira De Normas Técnicas, 1994.

ARMENZONI, M. et al. An integrated approach for demand forecasting and inventory management optimisation of spare parts. International Journal of Simulation and Process Modelling, v. 10, n. 3, p. 233, 2015. crossref

CHENG, Yung-Hsiang; YANG, Ann Shawing; TSAO, Hou-Lei. Study on Rolling Stock Maintenance Strategy and Spares Parts Management. [s. I.], p. 18, 2010.

COSTA, J. D. C. Gestão De Estoque De Materiais De Baixíssimo Giro Considerando Processos Críticos Para a Organização. p. 117, 2009.

COSTANTINO, F. et al. Spare parts management for irregular demand items. Omega (United Kingdom), v. 0, p. 1-10, 2017.

DE ALMEIDA, A. T. et al. Multicriteria and Multiobjective Models for Risk, Reliability and Maintenance Decision Analysis. [s.l: s.n.]. v. 231

DURÁN, O.; MACCHI, M.; RODA, I. On the relationship of spare parts inventory policies with Total Cost of Ownership of industrial assets. IFAC-PapersOnLine, v. 49, n. 28, p. 19-24, 2016.

GIANESI, I. G. N.; BIAZZI, J. L. Gestão estratégia dos estoques. Revista de Administração, [s. I.], v. 46, n. 3, p. 290-304, 2011. Disponível em:

<http://www.rausp.usp.br/busca/artigo.asp?num_artigo=1425> crossref

GHODRATI, B. Weibull and exponential renewal models in spare parts estimation: A comparison. International Journal of Performability Engineering, v. 2, n. 2, p. 135-147, 2006.

GODOY, D.; PASCUAL, R.; KNIGHTS, P. Critical Spare Parts Ordering Decisions Using Conditional Reliability and Stochastic Lead Time. Reliability Engineering \& System Safety. V.119, p.199-206. Elsevier, 2013. crossref

GOMES, A. V. P.; WANKE, P. Modelagem da gestão de estoques de peças de reposição através de cadeias de Markov. Gestao e Producao, v. 15, n. 1, p. 57-72, 2008. 
HU, Q. et al. OR in spare parts management: A review. European Journal of

Operational Research, v. 266, n. 2, p. 395-414, abr. 2018. crossref

KIRITSIS, D.; EMMANOUILIDIS, C.; KORONIOS, A. Engineering Asset Lifecycle

Management. London: Springer London, 2010. crossref

LEE, J; WANG, H.; New Technologies for Maintenance. Complex System

Maintenance Handbook. Springer series in reliability engineering, 2008.

O'CONNOR, PATRICK D. T.; KLEYNER, ANDRE. Practical reliability engineering. 5th Edition; John Wiley \& Sons, Ltd, 2012.

RIBEIRO, J. R. Sistemas construídos com tecnologia cots. 2007.

ROGERS, P.; RIBEIRO, K.; ROGERS, D. Gestão de Estoques, Risco, Método de Monte Carlo. VII SIMPOI FGVSP São Paulo, 2004.

ROGERS, P.; ROGERS, D.; RIBEIRO, K. Avaliando o risco na gestão financeira de estoques. VII SIMPOI FGV-SP, São Paulo, 2004.

SANTOS, M. R. DA S. Aplicação da Distribuição de Poisson para Determinação de Estoque Mínimo de Itens de MRO de Baixo Giro Aplicados em Manutenção de Ativos Industriais. [s.I.] Universidade Estadual Paulista. Faculdade de Engenharia, 2015.

SILVA FILHO, O. S.; CEZARINO, W.; RATTO, J. Planejamento agregado da produção: modelagem e solução via planilha Excel \& Solver. Revista Produção Online, v. 9, n. 3, p. 572-599, 2009. crossref

SOUZA, E. ÍNDICES DE CONFIABILIDADE DEVIDO A VEGETAÇÃO E PLANEJAMENTO DE PODAS DE ÁRVORES EM REDES DE DISTRIBUIÇÃO. [s.I.] Universidade Estadual Paulista "Júlio Mesquita Filho" Faculdade de Engenharia Câmpus de Ilha Solteira, 2013.

TEODORO FILHO, Alberto Magno et al. Modelo para determinar as quantidades em estoque de peças sobressalentes em uma planta de geração de energia. Revista Produção Online, Florianópolis, v. 17, n. 3, p. 828-856, set. 2017. ISSN 16761901. Disponível em:

<https://producaoonline.org.br/rpo/article/view/2549>. Acesso em: 05 jul. 2018. doi:https://doi.org/10.14488/1676-1901.v17i3.2549. crossref 
TURRINI, L.; MEISSNER, J. Spare parts inventory management: new evidence from distribution fitting. European Journal of Operational Research, 2017.

TURRIONI, J. B.; MELLO, C. H. P. Metodologia de pesquisa em engenharia de produção. Universidade Federal de Itajubá - UNIFEI, p. 191, 2012.

XENOS, H. Gerenciando a manutenção produtiva: o caminho para eliminar falhas nos equipamentos e aumentar a produtividade. 2. ed. [s.I.] Falconi Editora, 2012.

Recebido:16 Jun. 2018

Aprovado: 01 Mai. 2019

DOI: 10.3895/gi.v15n2.8569

Como citar:

SILVA, L.A; BRANDALISE N. Uso do método de Monte Carlo para determinação de custos associados aos estoques de sobressalentes para manutenção. R. Gest. Industr., Ponta Grossa, v. 15, n. 2, p. 96-113, Abr./Jun. 2018. Disponível em: <https://periodicos.utfpr.edu.br/rgi>. Acesso em: XXX.

Correspondência:

Lincon A. Silva

Programa de Pós-Graduação em Engenharia de Produção-Escola de Engenharia Industrial Metalúrgica de Volta Redonda-UFF, Av. dos Trabalhadores, 420, CEP: 27255-125, Volta Redonda, RJ, Brasil, e-mail: eng_lincon@hotmail.com

Nilson Brandalise

Programa de Pós-Graduação em Engenharia de Produção-Escola de Engenharia Industrial Metalúrgica de Volta Redonda-UFF, Av. dos Trabalhadores, 420, CEP: 27255-125, Volta Redonda, RJ, Brasil, e-mail: nilson_01@yahoo.com.br

Direito autoral:Este artigo está licenciado sob os termos da Licença Creative Commons-Atribuição 4.0 Internacional. 